\title{
Medición de la eficiencia y temperaturas en un transformador tipo seco que alimenta a un rectificador trifásico no controlado*
}

\section{José Antonio Álvarez-Salas Juan Segundo-Ramírez Víctor Manuel Cárdenas-Galindo Ricardo Álvarez-Salas}

Universidad Autónoma de San Luis Potosí

Facultad de Ingeniería

Av. Dr. Manuel Nava No. 8 Zona Universitaria

San Luis Potosí, S.L.P. C.P. 78290

MÉXICO

\author{
correos electrónicos (emails): \\ jaas@uaslp.mx \\ juan.segundo@uaslp.mx \\ vcardena@uaslp.mx \\ ralvarez@uaslp.mx
}

Recibido 31-01-2020, aceptado 15-05-2020.

\section{Resumen}

En este trabajo se presentan las pruebas que se realizaron en un laboratorio a un transformador tipo seco de capacidad de $5 \mathrm{kVA}$, el cual alimenta a un rectificador trifásico y una carga resistiva conectada en estrella. El propósito es evaluar el comportamiento de la eficiencia cuando en el transformador circulan corrientes no sinusoidales debido a la presencia del rectificador trifásico. Por medio de las mediciones de potencia se calculó la eficiencia en el transformador, de acuerdo a la Norma Oficial Mexicana NOM-002-SEDE/ENER-2014. Se presentan resultados y gráficas del comportamiento de la eficiencia. Con el rectificador trifásico en las terminales del transformador se produce una distorsión armónica de las corrientes en los devanados, lo que permitió analizar la modificación del factor $K$ a medida que se incrementó la carga resistiva en el rectificador. Adicionalmente se realizan mediciones de temperatura en el transformador mediante dos termopares que se encuentran incluidos entre los

*Este artículo es la versión extendida de los mejores trabajos del Congreso Nacional de Ingeniería Electromecánica y de Sistemas 2019 devanados y su núcleo, se presentan gráficas comparativas de las mediciones de temperatura.

Palabras clave: eficiencia, factor $K$, rectificador trifásico, temperatura, transformador tipo seco.

\section{Abstract \\ (Measurement of efficiency and temperatures in a dry type transformer that feeds a three-phase uncontrolled rectifier)}

This paper presents the laboratory tests conducted for a 5 kVA dry-type transformer feeding a wye-connected resistive load and a three-phase uncontrolled rectifier. The primary purpose is to evaluate the transformer efficiency performance against non-sinusoidal load currents. The laboratory tests are based on the standard NOM-002-SEDE/ENER-2014. The extensive conducted test results are reported through efficiency graphs. The variation of the harmonically distorted load current, due to the rectifier operation, allows to evaluate the $K$-factor variation for different load levels. In addition to this, the measured internal temperatures are presented in comparative graphs. The temperatures are obtained through thermocouples located between the core and the windings.

Index terms: efficiency, $K$-factor, three-phase rectifier, temperature, dry-type transformer.

\section{Introducción}

El amplio uso de dispositivos electrónicos en equipos que procesan la energía eléctrica en forma conmutada, genera una condición no sinusoidal de los voltajes y las corrientes que pudieran ser parte de las cargas en los transformadores de uso industrial. La presencia de voltajes y corrientes no sinusoidales en los conductores, equipos o elementos que conforman los sistemas eléctricos industriales, producen un incremento de sus pérdidas y la modificación del factor de potencia local o globalmente de la red [1].

Si los transformadores son uno de los dispositivos más empleados en los sistemas eléctricos industriales, su apropiado dimensionamiento determina la correcta operación del 
transformador. Típicamente las pérdidas presentes en los transformadores varían en función de su capacidad y tipo de cargas que se conectan a este; sin embargo, cuando se consideran cargas que son no sinusoidales se modifica la cantidad de pérdidas en el transformador [2].

Este artículo es la continuación de los trabajos previamente presentados en [3] y [4]. A diferencia de lo presentado en [3], en este artículo se analizan resultados experimentales de un transformador tipo seco de $5 \mathrm{kVA}$ que alimenta a un rectificador trifásico no controlado con carga resistiva.

Con un voltaje de alimentación sinusoidal en el transformador, la corriente será no sinusoidal cuando se conecta en las terminales una carga no lineal como lo es un rectificador trifásico. Las corrientes que circulan en los devanados son no sinusoidales, por lo que su valor eficaz se expresa por la ecuación [5]:

$$
I_{r m s}=\sqrt{I_{o}^{2}+I_{r m s-1}^{2}+I_{r m s-2}^{2}++\cdots I_{r m s-h}^{2}}
$$

Donde

$I_{0} \quad$ es la corriente promedio,

$I_{r m s-1}$ es la corriente eficaz de la componente fundamental,

$I_{r m s-h}$ es la corriente eficaz del $h$-armónica.

Un transformador en el que la corriente distorsionada excede el $5 \%$ del contenido armónico, puede producir un mayor calentamiento en sus devanados y núcleo [6].

La distorsión armónica total $(D A T)$ se emplea para cuantificar la presencia de armónicas en los voltajes y corrientes de los convertidores de potencia que se conectan a los sistemas eléctricos, se define para la corriente de acuerdo con la ecuación $[5]$ :

$$
D A T_{I}=\frac{\sqrt{I_{o}^{2}+I_{r m s-2}^{2}+I_{r m s-3}^{2}+I_{r m s-4}^{2} \cdots I_{r m s-h}^{2}}}{\sqrt{I_{r m s-1}^{2}}} 100
$$

Se realiza una ponderación entre los valores eficaces de los armónicos $I_{o}, I_{r m s-2}, I_{r m s-3}, \ldots, I_{r m s-h}$, y el valor eficaz de la componente fundamental $I_{r m s-1}$. Entre más distorsionada esté la forma de onda mayor será su DAT de corriente.

La valoración de las pérdidas en el transformador ante condiciones no sinusoidales, puede considerar un ajuste en la capacidad, así como una mejora en su eficiencia [6]. Las pérdidas en los transformadores pueden ser divididas en: pérdidas en vacío $P_{S C}$ y pérdidas con carga $P_{P C}$, como se indica en la ecuación [7]:

$$
P_{T}=P_{S C}+P_{P C}
$$

Las pérdidas con carga, son por calentamiento en los conductores debido al efecto Joule. La resistencia en corriente directa de los conductores y el valor eficaz de la señal no sinusoidal, contribuyen en la elevación de temperatura en las bobinas del transformador. Otra componente con carga son las pérdidas por corrientes circulantes y flujo disperso. Las pérdidas en vacío del transformador, en presencia de armónicos dependen del diseño del núcleo y la señal de voltaje [8].

Las pérdidas con carga en el transformador se calculan como se indica en la ecuación [9], [10]

$$
P_{P C}=P_{C U}+P_{C C}+P_{D}
$$

Donde

$P_{C U}$ son las pérdidas en el cobre

$P_{C C}$ son las pérdidas por corrientes circulantes

$P_{D}$ son las pérdidas por el flujo de dispersión

Las corrientes circulantes son muy pequeñas y son causadas por el flujo magnético, fluyen en el devanado, en el núcleo y en otras partes conductoras sujetas al campo magnético. Se incrementan con el cuadrado de la frecuencia de la corriente, por lo que es una componente significativa de las pérdidas en el transformador.

Las pérdidas en el cobre dependen del valor eficaz de la corriente de acuerdo con la siguiente ecuación:

$$
P_{C U}=I_{r m s}^{2} R_{c d}
$$

Donde

$I_{r m s}$ es el valor eficaz de la corriente no sinusoidal,

$R_{c d}$ es la resistencia en corriente directa de los devanados del transformador.

Las pérdidas por corrientes circulantes dependen principalmente del cuadrado del orden de armónico presente en las señales distorsionadas como se indica en la siguiente ecuación [6]:

$$
P_{C C=} N_{C C} \sum_{h=1}^{\infty} h^{2}\left(\frac{I_{r m s h}}{I_{r m s 1}}\right)^{2}
$$

Donde

$N_{c c}$ es una constante de proporcionalidad,

$I_{r m s 1}$ es el valor eficaz de la corriente fundamental,

$I_{r m s h}$ es el valor eficaz de las corrientes armónicas en por unidad referidas a la componente fundamental y $h$ es el orden del armónico. 
Las pérdidas por el flujo de dispersión son función de la geometría del núcleo y las bobinas, el nivel del voltaje del transformador, así como los materiales usados en la construcción del tanque. Este tipo de pérdidas se incrementan con la capacidad del transformador, en [9] se presenta un análisis detallado de este tipo de pérdidas para transformadores de distribución. En este trabajo se consideran despreciables.

Acorde a las normas asociadas con pruebas en los transformadores se tiene la NOM-002-SEDE/ENER-2014 [11], esta norma determina la eficiencia en los transformadores de distribución. La norma considera las pérdidas en vacío y debidas a la carga, referidas a un factor de carga del $80 \%$, derivadas de la medición de las pérdidas al $100 \%$ de la carga y corregidas a $85^{\circ} \mathrm{C}$ y un factor de potencia unitario de acuerdo con la ecuación:

$\% E=\frac{100 *\left(P * S_{\text {nom }} * 1000\right)}{\left(P * S_{\text {nom }} * 1000\right)+P_{\text {vacío }}+\left(P_{\text {carga }} * P^{2} * T\right)}$

Donde

$P \quad$ es la carga en por unidad (0.8),

$S_{\text {nom }}$ son los kVA nominales del transformador,

$P_{\text {vacio }}$ son las pérdidas de vacío a temperatura ambiente $(\mathrm{W})$,

$P_{\text {carga }}$ son las pérdidas debidas a la carga a temperatura de referencia a $85^{\circ} \mathrm{C}(\mathrm{W})$,

$T$ es un factor de corrección para las pérdidas a $70^{\circ} \mathrm{C}$ (0.952332).

El factor $K$ mide los efectos del calentamiento provocado por las corrientes armónicas, en relación con la capacidad de la carga y las pérdidas del transformador [6], [12]:

$$
K=\sum_{1}^{\infty} h^{2}\left(\frac{I_{h}}{I_{r m s}}\right)^{2}
$$

Donde

$I_{h}$ es la corriente armónica,

$I_{r m s}$ es la corriente eficaz total,

$h$ es el orden del armónico.

El factor $K$ de un transformador es un indicador de la capacidad que tiene el transformador para entregar potencia a una carga no lineal, sin exceder el límite de temperatura para la cual fue diseñado. El factor $K=1$ indica que no existen armónicas y se asocia a las cargas lineales. Cuanto mayor sea el factor $K$ mayor será el efecto de calentamiento en el transformador por la presencia de armónicas en sus señales de voltaje y corriente. El factor $K$ es utilizado por los fabricantes y usuarios, para ajustar el nivel de carga en el transformador como función de las corrientes armónicas distorsionadas debidas al uso de convertidores de potencia [12], [13].

\section{Desarrollo}

El propósito del proyecto es analizar el comportamiento de la eficiencia, cambio del factor $K$ y la elevación de temperaturas, en un transformador tipo seco, el cual se utiliza típicamente para procesos de rectificación industrial. El prototipo se encuentra en el Laboratorio de Máquinas Eléctricas del Área Mecánica y Eléctrica de la Facultad de Ingeniería. Conforma parte de la infraestructura que se tiene disponible para el estudio de transformadores.

En la figura 1 se muestra el transformador en su gabinete y la distribución de las bobinas del transformador. El transformador tiene un arreglo en delta en el primario de 220V/13.2 A y un arreglo en estrella en el secundario de $440 \mathrm{~V} / 6.56 \mathrm{~A}$. Cuenta con derivaciones para realizar ajustes al nivel de voltaje del secundario desde $440 \mathrm{~V}$ a $460 \mathrm{~V}$, para las pruebas de eficiencia se conectaron estas derivaciones para un voltaje de $440 \mathrm{~V}$. En la tabla 1 se muestran los valores nominales del transformador.

El fabricante incluyó en las bobinas y núcleo del transformador termopares del tipo $J$, el propósito fue tener un registro de la

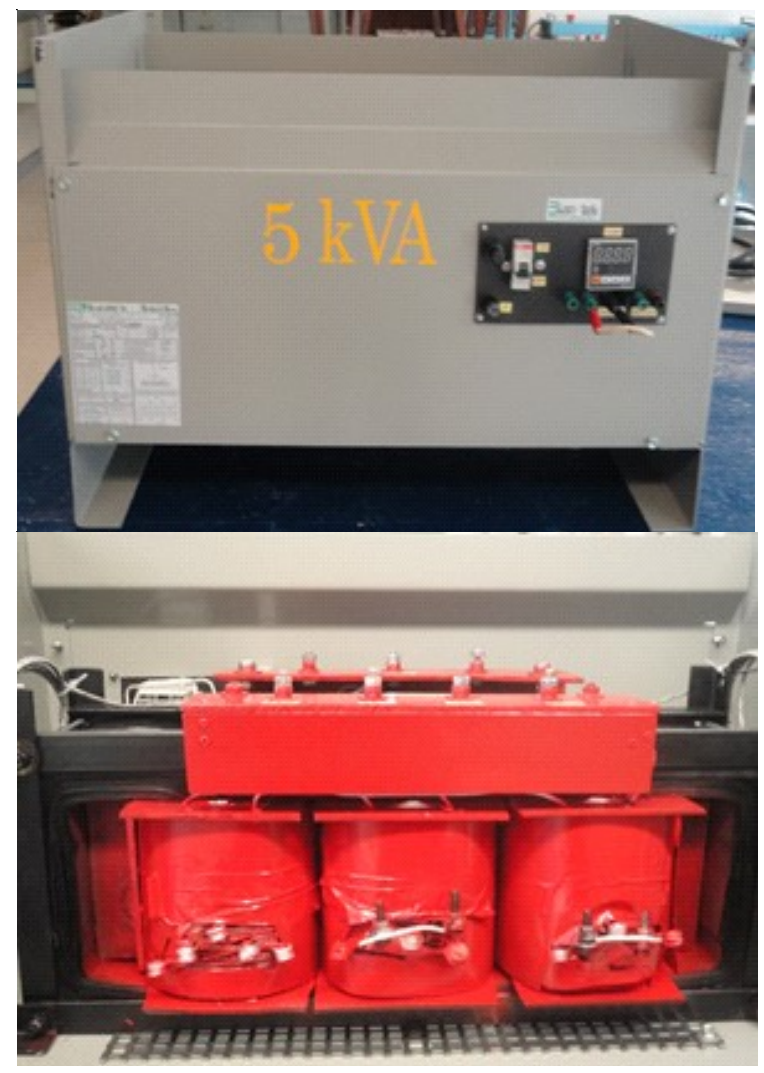

Fig. 1. Gabinete y bobinas del transformador de 5 kVA. 
Tabla 1. Valores nominales del transformador de $5 \mathrm{kVA}$.

\begin{tabular}{ccccc}
\hline Fases & Capacidad & Tipo & Frecuencia & \% Eficiencia \\
3 & $5 \mathrm{kVA}$ & Seco & $60 \mathrm{~Hz}$ & 96 \\
\hline Voltaje & Corriente & Conexión & $\%$ Impedancia & Elev.-Temp. \\
$220 \mathrm{~V} / 440 \mathrm{~V}$ & $13.2 \mathrm{~A} / 6.56 \mathrm{~A}$ & Delta-Estrella & 3.75 & $65^{\circ} \mathrm{C}$ \\
\hline
\end{tabular}

temperatura interna del transformador. En la figura 2 aparecen las imágenes de las conexiones de termopares y del indicador para el registro de las temperaturas en el transformador. El registro se llevó a cabo en intervalos de 10 minutos hasta los 60 minutos de operación. Las pruebas del transformador se realizaron con porcentajes de carga, en cada etapa de prueba se requirió de 120 a 160 minutos para un proceso de enfriamiento.

Se utilizó un analizador de calidad de la energía trifásico [12], considerando que se debe registrar señales no sinusoidales de voltaje y corriente en el transformador cuando se utiliza el rectificador. Con los sensores de voltaje y corriente del analizador en las terminales del primario y secundario, se procesan las mediciones de potencia que requiere la ecuación (7) para el cálculo de la eficiencia. En la figura 3 se indican los diagramas de conexión en el transformador con la carga resistiva y el rectificador trifásico.

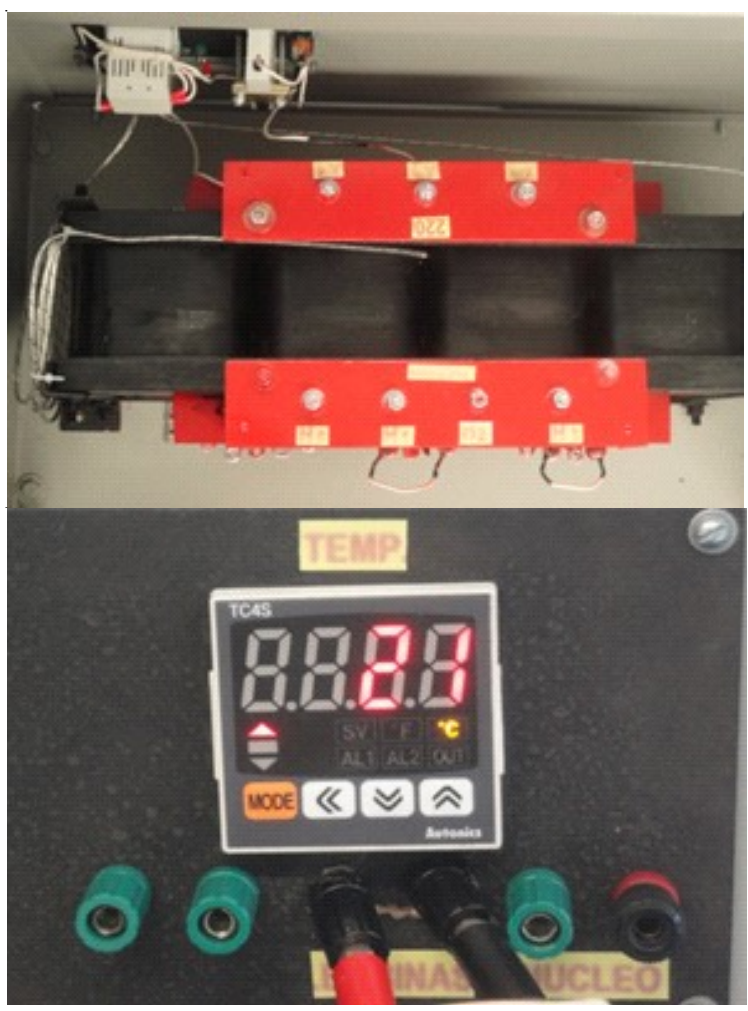

Fig. 2. Termopares en las bobinas y núcleo del transformador.
En la figura 4, se muestra la estructura interna del transformador con el arreglo de las resistencias de potencia como carga. La carga resistiva se conectó en estrella, con porcentajes de: $80 \%, 100 \%$ y $120 \%$. El valor nominal del banco de resistencias de potencia es de: $16 \Omega @ 1000 \mathrm{~W} / 127 \mathrm{~V}$.

En la figura 5 se muestra el transformador con el rectificador y el arreglo de resistencias de potencia. El rectificador trifásico no controlado se construyó con diodos de potencia de: 400$1000 \mathrm{~V} @ 15$ A en un arreglo de dos diodos y sus disipadores por cada rama del convertidor. A la salida del rectificador se produce una corriente directa con una salida de $570 \mathrm{~V}$ y $8.5 \mathrm{~A}$ que alimenta a una carga resistiva.

\section{Resultados}

Los resultados para el cálculo de la eficiencia y el factor $K$ se realizaron con la carga resistiva y el rectificador trifásico. Las mediciones con los circuitos de prueba y cambios en los porcentajes de carga se indican en las tablas 2 y 3 . El nivel de la distorsión armónica total de las corrientes del transformador, fue el indicador de la presencia de armónicas por la conexión del rectificador al transformador. En una señal de corriente sin contenido armónico la distorsión armónica tiene un valor nulo, de acuerdo a la ecuación 2. Sin embargo, en las mediciones con carga resistiva el voltaje de la fuente trifásica presenta
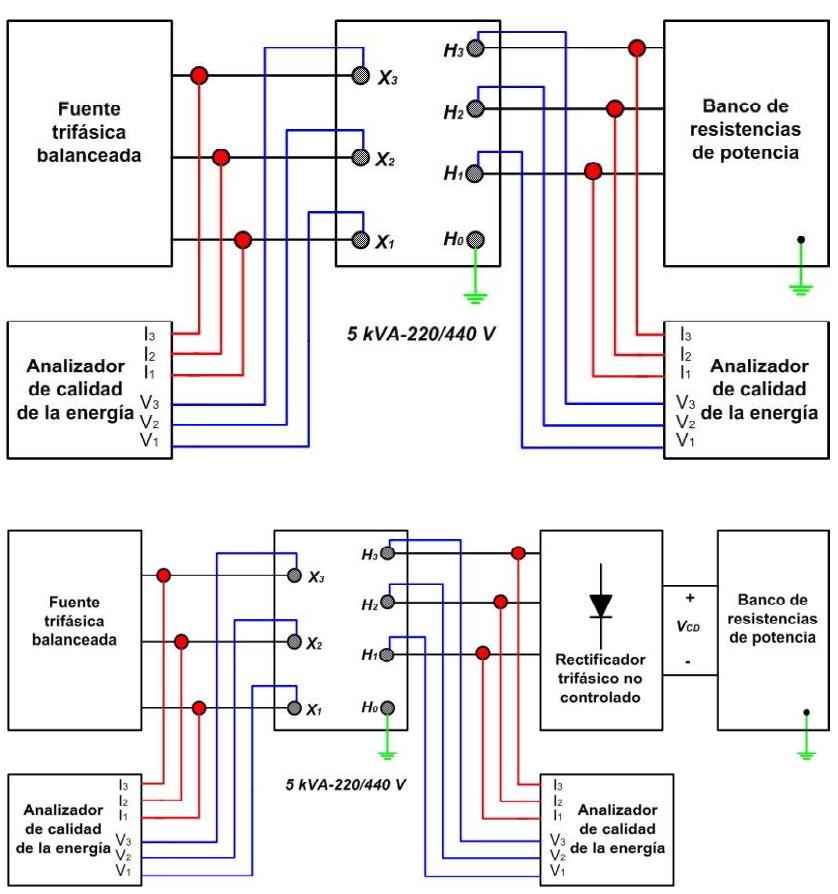

Fig. 3. Diagrama de conexiones en el transformador para las mediciones de potencia. 

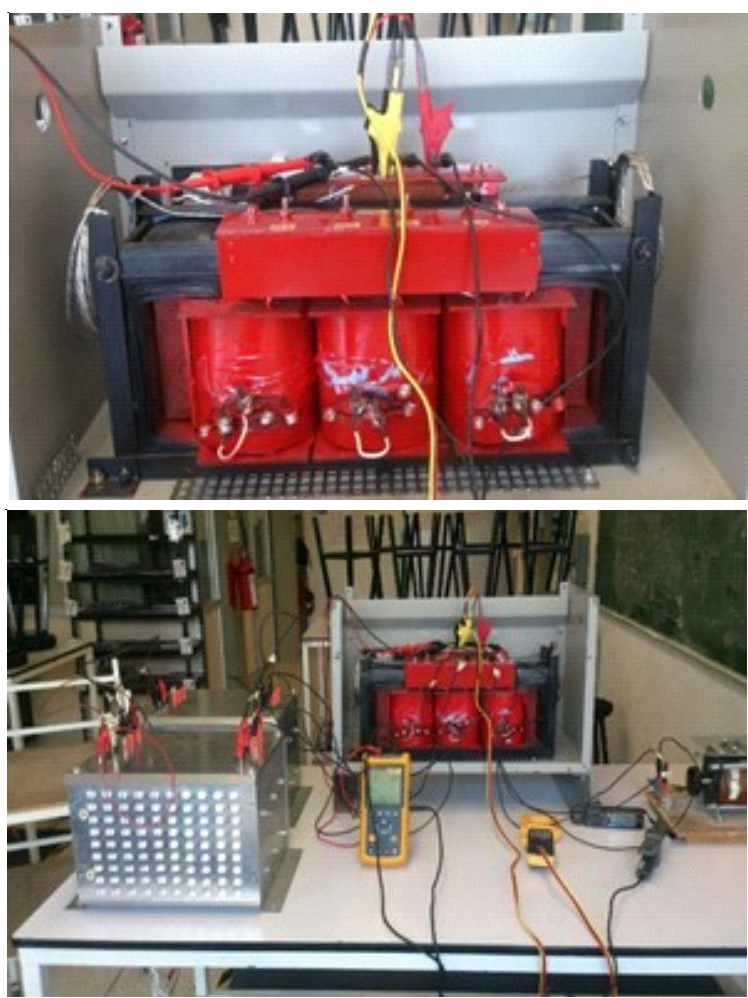

Fig. 4. Mediciones en el transformador con carga resistiva.

Tabla 2. Distorsión armónica y potencias del transformador con el circuito resistivo.

\begin{tabular}{ccccc}
\hline $\begin{array}{c}\text { Carga } \\
(\%)\end{array}$ & $\begin{array}{c}\text { DAT-Ip } \\
(\%)\end{array}$ & $\begin{array}{c}\text { DAT-Is } \\
(\%)\end{array}$ & $\begin{array}{c}\text { Pent } \\
(\mathbf{k W})\end{array}$ & $\begin{array}{c}\text { Psal } \\
(\mathbf{k W})\end{array}$ \\
\hline 80 & 0.8 & 0.8 & 4.03 & 3.89 \\
100 & 0.9 & 0.8 & 5.05 & 4.84 \\
120 & 0.9 & 0.8 & 6.05 & 5.75 \\
\hline
\end{tabular}
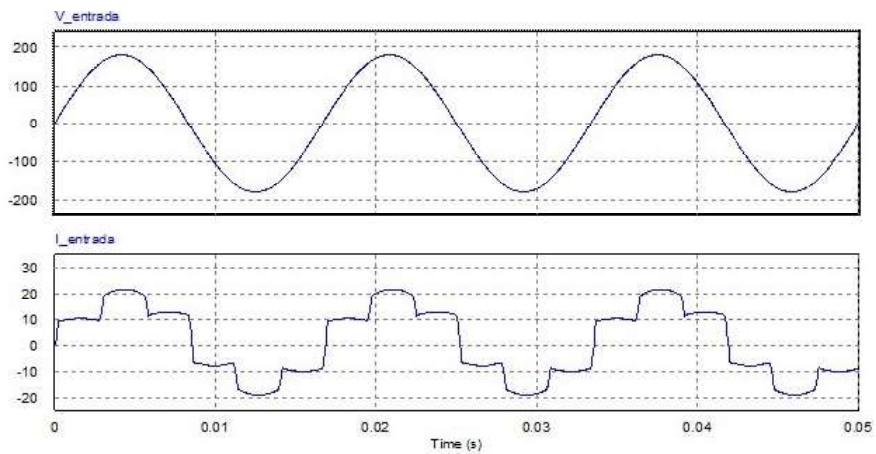

Fig. 6. Formas de onda de voltaje y corriente a la entrada en el transformador con el rectificador trifásico.

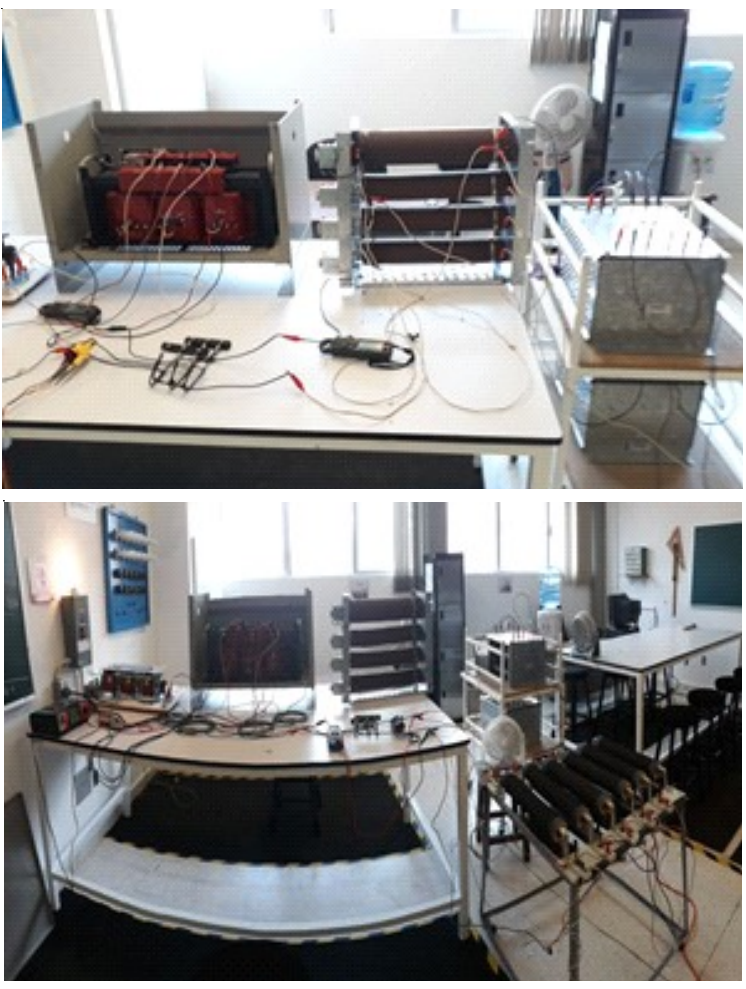

Fig. 5. Mediciones en el transformador con el rectificador trifásico y carga resistiva.

Tabla 3. Distorsión armónica y potencias del transformador con el rectificador trifásico.

\begin{tabular}{ccccc}
\hline $\begin{array}{c}\text { Carga } \\
(\%)\end{array}$ & $\begin{array}{c}\text { DAT-Ip } \\
(\%)\end{array}$ & $\begin{array}{c}\text { DAT-Is } \\
(\%)\end{array}$ & $\begin{array}{c}\text { Pent } \\
(\mathbf{k W})\end{array}$ & $\begin{array}{c}\text { Psal } \\
(\mathbf{k W})\end{array}$ \\
\hline 80 & 24.80 & 27.50 & 4.38 & 4.12 \\
100 & 25.00 & 27.20 & 5.09 & 4.65 \\
120 & 25.50 & 27.56 & 6.06 & 5.41 \\
\hline
\end{tabular}

una pequeña distorsión, por lo que en la tabla 2 la distorsión armónica de la corriente presenta valores diferentes de cero.

En las figuras 6 y 7, se muestran las señales de simulación digital del voltaje y la corriente en la entrada y salida del transformador con el rectificador trifásico. Las formas de onda de corriente tienen una distorsión armónica del 25 al 27\%, un valor típico que se asocia con las corrientes armónicas de un sistema de rectificación trifásico. Para la simulación digital de los voltajes y las corrientes, en el modelo del transformador se utilizaron 

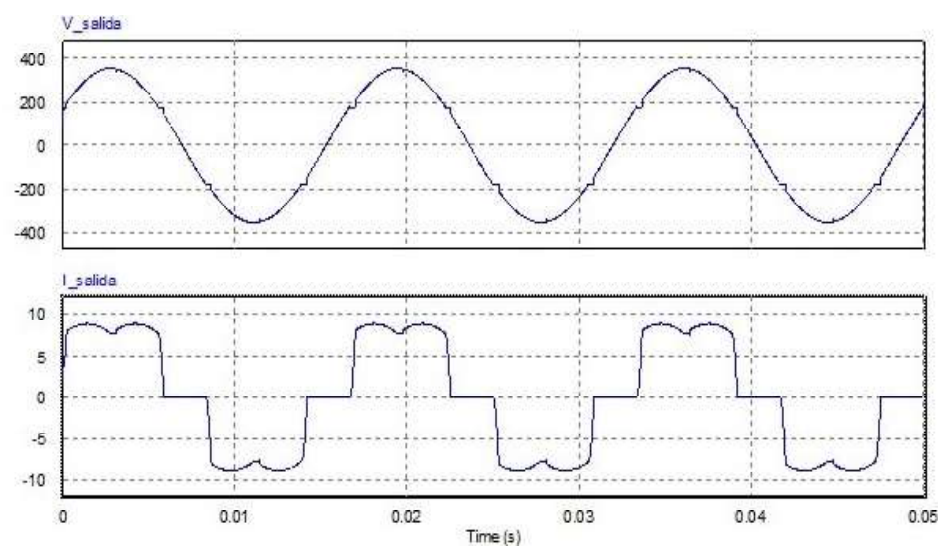

Fig. 7. Formas de onda de voltaje y corriente a la salida del transformador con el rectificador trifásico. los parámetros de resistencias y reactancias que se obtuvieron de las pruebas indicadas en el anexo.

La eficiencia del transformador se calculó con la ecuación (7) empleando las mediciones de las tablas 2 y 3 así como la medición de la potencia en vacío de aproximadamente de $50 \mathrm{~W}$. Con la información anterior se construyeron las gráficas de la figura 8 en donde se muestra la modificación de la eficiencia del transformador con la carga resistiva y el rectificador trifásico.

El decremento en la eficiencia del transformador, representa principalmente un incremento de las pérdidas en el cobre y por corrientes circulantes, las cuales se indican en las ecuaciones (5) y (6). A valor nominal y carga resistiva el transformador presenta una efi-
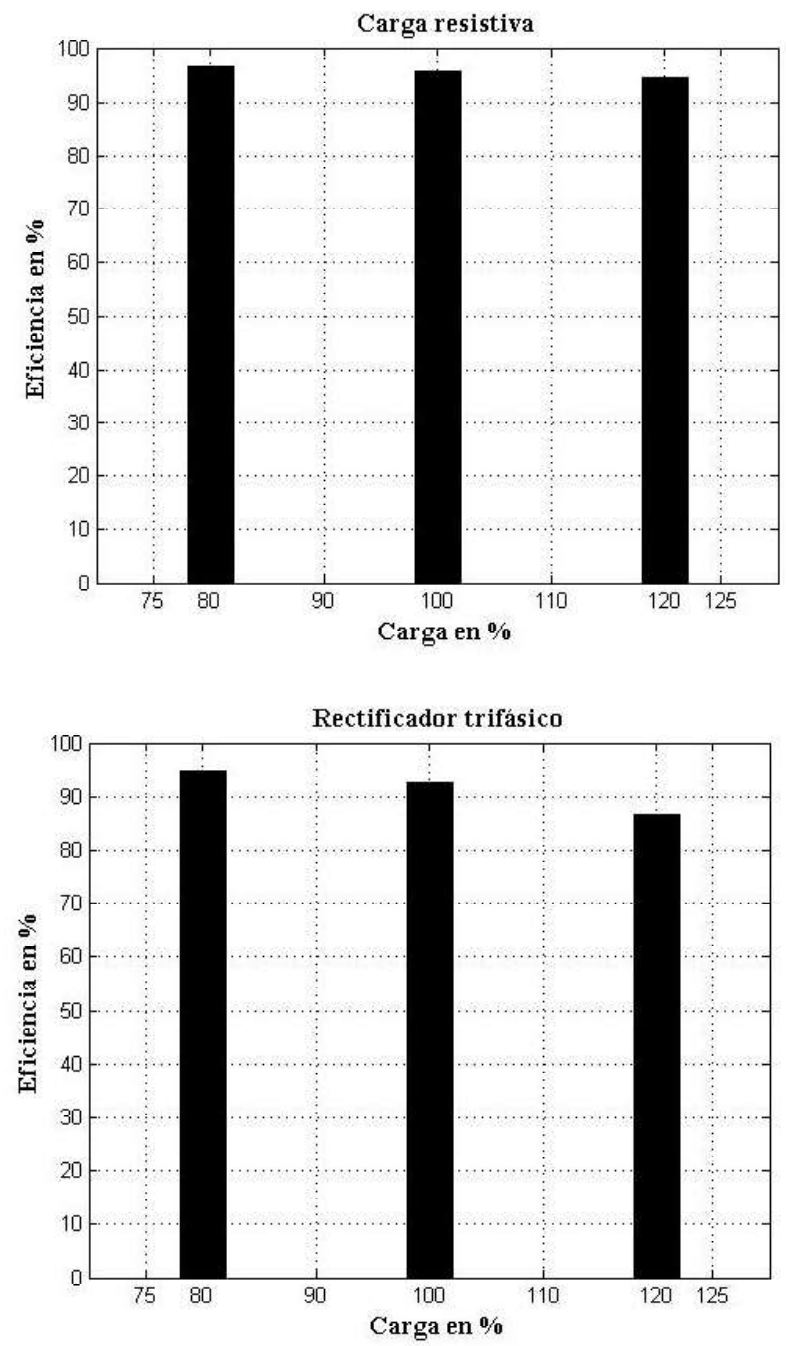

Fig. 8. Comportamiento de la eficiencia del transformador.
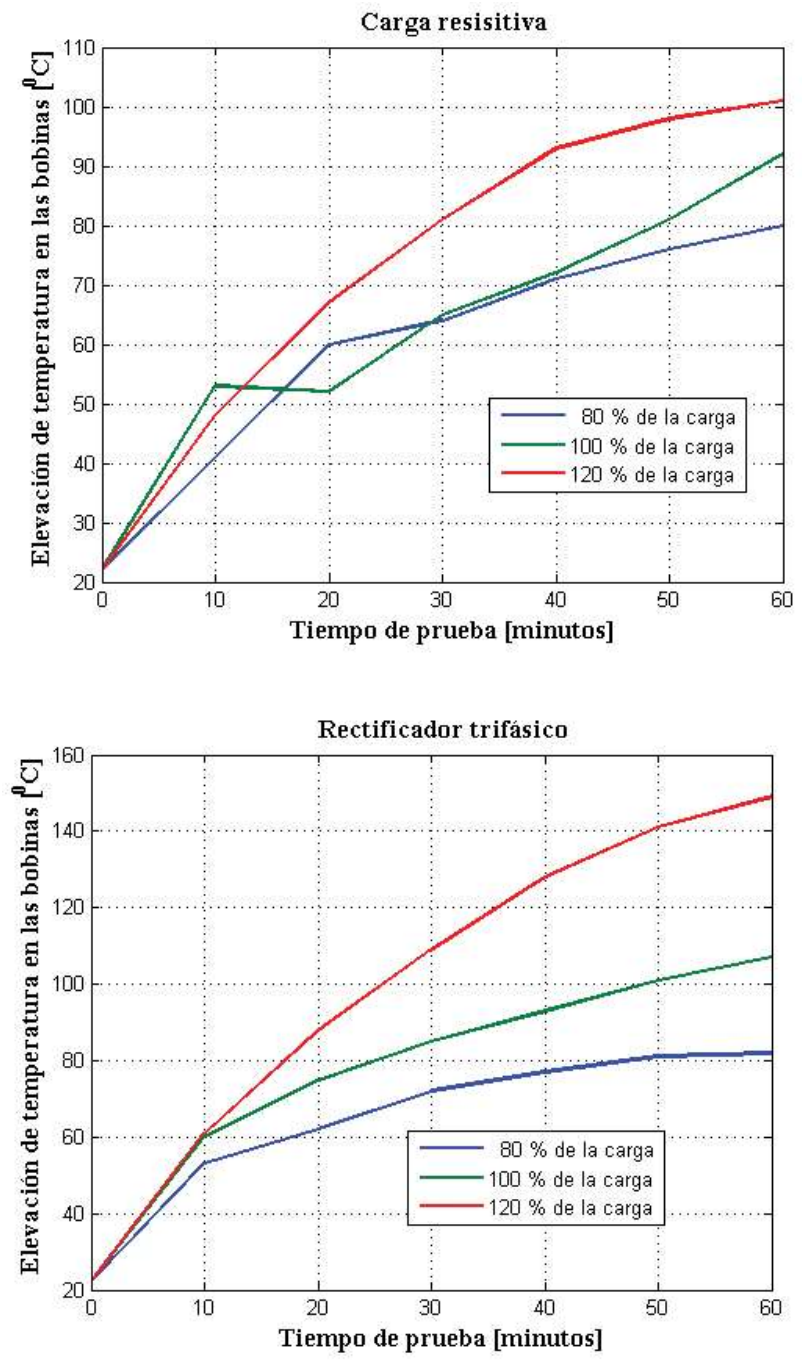

Fig. 9. Elevación de temperatura en las bobinas del transformador. 
ciencia del 95.6\%, mientras que con el rectificador dismunyó a $92.5 \%$. Con un incremento del $20 \%$ en la carga resistiva del transformador su eficiencia disminuyó a $94.5 \%$, y con el rectificador disminuyó al 88.8\%. La sobrecarga y la no linealidad de la corriente afectan la eficiencia del transformador de forma significativa, por lo que el transformador limita la capacidad de entregar los $5 \mathrm{kVA}$ que se especifican en su placa.

En el proceso de medición de las temperaturas en las bobinas y núcleo, se realizó un registro de lecturas a través del indicador conectado a los termopares y colocado en la parte frontal del gabinete del transformador. Con estos registros, se construyeron las gráficas de incremento de temperaturas en las bobinas como se indica en la figura 9. De acuerdo con sus datos nominales, el transformador tiene la posibilidad de permitir una elevación de temperatura de $65^{\circ} \mathrm{C}$ sobre el medio ambiente en el que se encuentre.
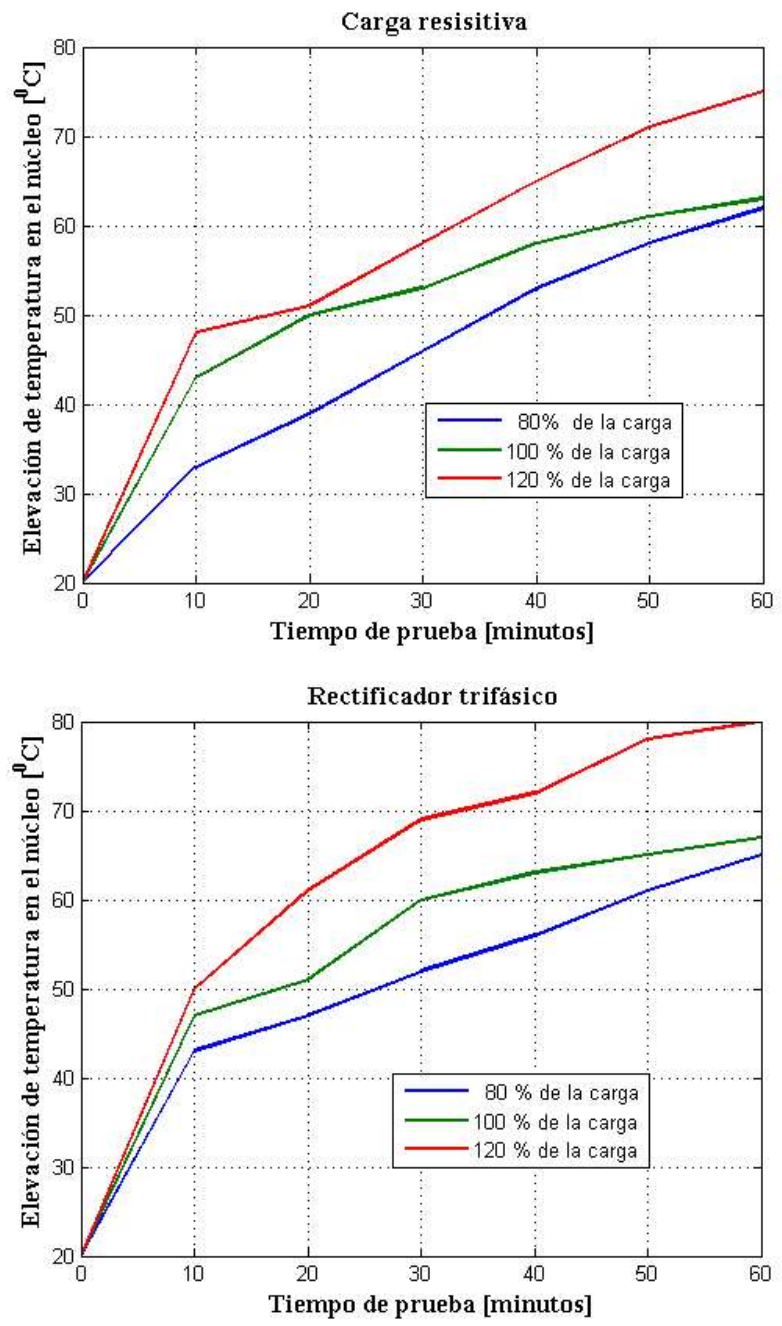

Fig. 10. Elevación de temperatura en el núcleo del transformador.
En la figura 9 donde aparece la gráfica del rectificador trifásico con sobrecarga, se muestra un incremento mayor a $\operatorname{los} 40^{\circ} \mathrm{C}$ en las bobinas, respecto a las pruebas con carga resistiva. El transformador no cuenta con ventilación externa, por lo que existe una concentración de calor dentro del gabinete, cada prueba fue realizada con intervalos de enfriamiento para tener una misma temperatura de referencia ante los cambios de carga.

En la figura 10, se indica el incremento de temperatura en el núcleo con la carga resistiva y el rectificador. En estas dos gráficas de puede observar que existe proximidad en las curvas, lo anterior debido a que las pérdidas en el núcleo son función principalmente de la señal de voltaje. Para el caso de los voltajes en los devanados del transformador con carga resistiva y con el rectificador son prácticamente sinusoidales.

Con las mediciones de las corrientes en el analizador de calidad de la energía, se obtuvieron las magnitudes y frecuencias de las corrientes armónicas debidas al rectificador. Empleando la ecuación (8) y las mediciones de las corrientes se calcularon los diferentes valores del factor $K$ que aparecen en la tabla 4. En un transformador con alimentación sinusoidal y carga lineal su factor es $K=1$.

Debido a una pequeña distorsión en la señal de voltaje que alimenta al transformador con carga resistiva, el factor $K$ de la tabla 4 no tiene un valor exacto de la unidad. Para el caso del rectificador trifásico con la DAT $=27 \%$ en la corriente, el factor $K$ se incrementó cuatro veces independientemente del porcentaje de carga. De acuerdo con la ecuación (6), el cuadrado del orden de las armónicas de corriente de frecuencias superiores, contribuyen al incremento del factor. Por lo anterior se valida que el factor $K$ es dependiente de la distorsión armónica de sus variables eléctricas como se indica en [6] y [15].

El incremento de las pérdidas en los transformadores, requiere de un sistema de monitoreo y diagnóstico que permita identificar las posibles fallas por las sobrecargas o efecto de las armónicas que circulan en sus devanados. En los trabajos que se presentan en [16] y [17], se hace referencia a técnicas o sistemas de diagnóstico y monitoreo aplicado a transformadores industriales y de potencia.

Tabla 4. Factor $K$.

\begin{tabular}{cccc}
\hline Carga resistiva & Factor $\boldsymbol{K}$ & Rectificador trifásico & Factor $\boldsymbol{K}$ \\
\hline Prueba @ $80 \%$ & 1.004 & Prueba @ $80 \%$ & 4.281 \\
Prueba @ $100 \%$ & 1.005 & Prueba @ $100 \%$ & 4.150 \\
Prueba@ $100 \%$ & 1.006 & Prueba @ $120 \%$ & 4.540 \\
\hline
\end{tabular}




\section{Conclusiones}

En este trabajo se probó experimentalmente la reducción de la eficiencia e incremento del factor $K$ para un transformador trifásico del tipo seco, que alimenta a un rectificador trifásico no controlado. El cálculo de la eficiencia se realizó a través de la Norma Oficial Mexicana NOM-002-SEDE/ENER-2014. El cálculo del factor $K$ se realizó a través del estándar IEEE-C57.1101988. La circulación de las corrientes armónicas en los devanados del transformador, tuvieron implicación en la elevación de temperatura dentro del transformador. Lo anterior se verificó con las mediciones que se realizaron a través de los termopares incluidos en las bobinas y en el núcleo, así como con los cambios de carga en las terminales del transformador. Las normas vigentes son claras al respecto de la conexión de convertidores electrónicos de potencia con los transformadores de uso industrial. Analizar el comportamiento de la eficiencia y factor $K$ en los transformadores permite a estudiantes e ingenieros la especificación correcta de estos equipos. Este trabajo es una contribución a la infraestructura que fortalece el desarrollo de prácticas en transformadores, tanto para los programas de licenciatura como de posgrado en Ingeniería Eléctrica dentro de la Facultad de Ingeniería.

\section{Agradecimientos}

Los autores agradecen el apoyo recibido de los laboratorios involucrados de la Facultad de Ingeniería a través de su personal, alumnos e infraestructura en las pruebas realizadas al transformador de $5 \mathrm{kVA}$.

\section{Anexo}

En la tabla 5 aparecen los parámetros del circuito equivalente del transformador. Cuando se recibió el transformador, se realizaron las pruebas de circuito abierto y circuito corto de acuerdo a lo que se especifica en [18], [19]. En estas pruebas se verificó la condición del transformador. Con las mediciones se procedió a obtener los valores de las resistencias y reactancias que aparecen en la tabla, los cuales se encontraron acorde a su capacidad.

Tabla 5. Parámetros del transformador de 5 kVA.

\begin{tabular}{ccc}
\hline $\begin{array}{c}\text { Impedancia del } \\
\text { primario }\end{array}$ & $\begin{array}{c}\text { Impedancia del } \\
\text { secundario }\end{array}$ & $\begin{array}{c}\text { Rama de } \\
\text { magnetización }\end{array}$ \\
$R_{1}=0.13 \Omega$ & $R_{2}=0.515 \Omega$ & $X_{M}=266.310 \Omega$ \\
$X_{1}=0.04 \Omega$ & $X_{2}=0.160 \Omega$ & $R_{C}=565.102 \Omega$ \\
\hline
\end{tabular}

\section{Referencias}

[1] L. Astudillo, A. Vásquez, J. Rojas, "Implementación de un filtro pasivo para compensación armónica y corrección del factor de potencia," Científica, vol 20, núm. 1, pp 3-11, enero-junio 2016.
[2] IEEE Std. C57.120-2017, Guide for Loss Evaluation of Distribution and Power Transformers and Reactors, 2017.

[3] I. G. Perez, J. A. Alvarez, J. Segundo, "Comparación de las pérdidas en un transformador tipo seco de $5 \mathrm{kVA}$ ante carga lineal y no lineal," Revista Pistas Educativas, vol. 40, núm. 130, 2018.

[4] J. A. Alvarez, J. Segundo, V. M. Cardenas, R. Alvarez, "Medición de la eficiencia y temperaturas en un transformador seco que alimenta un rectificador $3 \varphi "$, XVIII Congreso Nacional de Ingeniería Electromecánica y de Sistemas (CNIES 2019), Ciudad de México, Nov 2019.

[5] IEEE Std. 1459-2010, Definitions for the Measurement of Electrical Power Quantities Under Sinusoidal, Non-sinusoidal, Balanced, Unbalanced Conditions, 2010.

[6] IEEE Std. C57.110-1988, Recommended Practice for Establishing Transformer Capability When Supplying Non-Sinusoidal Load Currents, 1988.

[7] A. Elmoudi, M. Lethonen, "Effect of harmonics on transformers loss of life," IEEE International Symposium on Electrical Insulation, pp. 408-411, 2006.

[8] R. Arseneau, E. So, E. Hanique, "Measurments and Correction of No-Load Losses of Power Transformers," IEEE Transactions on Instrumentation and Measurement, vol. 54, núm. 2, pp 503506, 2005.

[9] J. C. Olivares, J. Cañedo, P. Moreno, J. Driesen, R. Escarela S. Palanivasagam, "Experimental study to reduce the distributiontransformers stray losses using electrto-magnetic shields," Electric Power Systems Research, vol. 63, 2002, pp. 1-7.

[10] E. Cazacu, L. Petrescu, V. Lonita, "Losses and temperature rise within power transformers subjected to distorted currents," 15th International Conference on Electrical Machines Drives and Power Systems, 2017.

[11] Norma Oficial Mexicana NOM-002-SEDE/ENER-2014, Requisitos de seguridad y eficiencia energética para transformadores de distribución, 2014.

[12] S. Hasan, I. Daut, S. Taib, "K-Factor due to Implementing of DC Unbalanced/Balanced of a Transformer", 2007 Asia-Pacific Conference on Applied Electromagnetics Procedings, Decembre 4-6, 2007, Melaka, Malaysia.

[13] T. Shun, X. Xiangning "Comparing transformer de-rating computed using the harmonic loss factor FHL and K-Factor", 3rd International Conference on Electric Utility Deregulation and Restructuring and Power Thechnologies, 2008, pp. 16311634.

[14] Metrel Power Quality Analyzer MI2192, Instruction Manual Version 3, Code No. 20750701.

[15] M. Salih Taci, A. Domijan Jr. "The Effects of Linear and Nonlinear Operation Modes in Transformers", 2004 11th Int. Confernce on Harmonics and Quality of Power, 2004, pp. 244-249.

[16] M.C. Hernandez, J. Hernandez, F.E. Hernandez, "Sistemas para la gestión de la condición de los transformadores de potencia," Científica, vol 17, núm. 2, pp. 83-88, abril-junio 2013.

[17] R.S. Bhide, M.S. Srinivas, I. Voloh, " Detection of inter-turn fault in transformers at incipient level," XXI Int. Conference on Electrical Machines (ICEM), septiembre 2014, pp, 1542-1548

[18] V.Perez-Amador, Pruebas de equipo eléctrico, transformadores de distribución y potencia, Limusa, 1985.

[19] B. S. Guru, H. R. Hiziroglu, Máquinas eléctricas y transformadores, Alfaomega, $3^{\mathrm{a}}$ ed., 2002. 
